\title{
Erosão dentária na dentição permanente: epidemiologia e diagnóstico
}

\author{
Dental erosion in permanent dentition: epidemiology and diagnosis
}

\author{
Mabel Miluska Suca Salas* \\ Luiz Alexandre Chisini** \\ Fabiana Vargas-Ferreira** \\ Flávio Fernando Demarco ${ }^{* * *}$
}

\section{Resumo}

Objetivo: o presente estudo literário revisou e analisou estudos epidemiológicos, realizados em crianças de 8 a 19 anos com dentição permanente, sem tratamentos ortodônticos ou problemas sistêmicos, e que tenham avaliado possíveis fatores comumente associados à erosão. Revisão de literatura: a erosão dentária (ED) é definida como a perda de tecido duro da superfície dental dada por influência química sem envolvimento bacteriano. É uma condição bucal multifatorial associada a fatores extrínsecos, como a ingestão de bebidas carbonatadas e ácidas, ou intrínsecos, como a associação a episódios de regurgitação, que resultam na desmineralização da superfície dentária. O desgaste por erosão é comumente associado a outros tipos de desgastes dentários de diferentes etiologias. A erosão dentária pode promover problemas estéticos, funcionais e a alteração na oclusão quando não controlada. Considerações finais: a prevalência em crianças com dentição permanente variou de $7,2 \%$ a $55.5 \%$, e os resultados apontam que a maior prevalência de erosão dentária está associada com a maior idade e o maior consumo de alguns alimentos ácidos, como os refrigerantes, e com o menor consumo de alguns alimentos lácteos, como o leite. Em crianças e adolescentes, a severidade das lesões foi limitada ao esmalte. A associação a alguns fatores socioeconômicos e biológicos demonstrou resultados divergentes.

Palavras-chave: Erosão dentária. Criança. Adolescentes. Prevalência. Fatores associados.

\section{Introdução}

A erosão dentária foi ganhando importância como um problema de maior reconhecimento por parte da comunidade científica e clínica, em decorrência do aumento da incidência que está sendo observada em crianças e adolescentes ${ }^{1,2}$. Estudos têm observado a existência de possíveis fatores que se encontram presentes durante o processo de aparecimento da erosão, de forma pouco conclusiva ${ }^{3}$. A erosão dentária é caracterizada pela perda irreversível e cumulativa do tecido dentário, que pode ser agravada com o tempo, devido à interação com outros tipos de desgaste ${ }^{4}$. O desgaste erosivo severo pode causar perda funcional e estética ${ }^{5}$. A erosão pode estar associada com hipersensibilidade, alteração na oclusão dentária e exposição da polpa ${ }^{6}$.

Existe dificuldade para o reconhecimento dos sinais iniciais do desgaste dentário por erosão e o entendimento da localização das populações em risco devido às diversas variáveis associadas à erosão. O diagnóstico clínico precoce é fundamental e é baseado, primariamente, no estudo da dieta e na frequência da ingesta de alimentos e bebidas ácidas. Estudos observam a existência de fatores que estão presentes durante o processo de aparecimento da erosão. A dieta constituída pelo consumo de bebidas ou comidas ácidas, como os refrigerantes, os su-

\footnotetext{
Doutoranda em Odontologia, Universidade Federal de Pelotas, Brasil.

Mestrando em Odontologia, Universidade Federal de Pelotas, Brasil.

** Pós-doutoranda, Ulbra, doutora em Epidemiologia, Universidade Federal de Pelotas, Brasil.

*** Professor titular de Odontologia e Epidemiologia, Universidade Federal de Pelotas, Brasil.
} 
cos de frutas cítricas, além do uso de drogas ácidas, e a influência da temperatura das frutas ácidas ${ }^{7}$, destacando-se o suco de maçã, relatado na pesquisa de Okunseri et al. ${ }^{8}$ (2011), podem estar diretamente associados à erosão dentária ${ }^{8,9}$.

O nível socioeconômico apresenta influência contraditória. Alguns estudos não encontraram relação com o desgaste dentário ${ }^{10}$, outros estudos observaram crianças de baixo nível econômico com maior prevalência de erosão ${ }^{1}$. A escolaridade dos pais mostrou-se um fator associado, e estabeleceu-se que quanto maior a escolaridade dos pais, menor a prevalência evidenciada nas crianças ${ }^{9}$. O tipo de escola também foi abordado como um fator associado, resultando que nas escolas particulares, a erosão foi mais prevalente em relação às escolas públicas ${ }^{11}$. Crianças com cárie tiveram maior prevalência de erosão dentária em relação às crianças sem cárie ${ }^{12}$.

O conhecimento das características da erosão dentária facilita o diagnóstico dessa condição. $\mathrm{O}$ diagnóstico precoce é parte importante do tratamento em relação à erosão, pois torna possível a implementação de medidas preventivas logo no início. Isso diminui a chance da necessidade de tratamentos restauradores extensos ${ }^{4}$. A prevenção se dá a partir do conhecimento e reconhecimento por parte dos profissionais da odontologia dos fatores que predispõem o indivíduo à erosão dentária. $\mathrm{O}$ objetivo da presente revisão foi analisar a epidemiologia e as características clínicas da erosão dentária na dentição permanente de crianças e/ou adolescentes.

\section{Revisão da literatura}

\section{Conceito}

A erosão dentária é definida como a perda de tecido duro da superfície dental ${ }^{13}$. A estrutura dentária é quimicamente retirada do elemento dental por ação de ácidos, sem o envolvimento de bactérias $^{9}$. Usualmente é observada interagindo com outros tipos de desgaste de diferentes etiologias como abrasão e atrição ${ }^{4,13}$.

\section{Etiologia}

Os ácidos podem ser de dois tipos: extrínsecos e intrínsecos. Ácidos extrínsecos estão relacionados ao consumo exagerado e contínuo de alimentos e bebidas ácidas. Ácidos intrínsecos são provenientes do ácido gástrico, como consequência de refluxos ${ }^{14}$. Quando a lesão é ocasionada pelo ácido do tipo extrínseco, tende a ser na região da face vestibular ${ }^{15}$. Se os ácidos são de origem intrínseca, as lesões tendem a se localizar nas regiões das faces palatinas e oclusais $^{15}$.

\section{Processo de desmineralização}

A lesão de erosão é caracterizada pela desmineralização da camada superficial do esmalte com a subsequente perda da estrutura dental a partir do contato frequente com ácidos. Íons de cálcio e fosfato são removidos, deixando espaços vazios, micrométricos, nos tecidos duros do dente. Isso gera o aparecimento de uma camada superficial fragilizada e desmineralizada ${ }^{14}$.

Com a camada superficial fragilizada, ocorre um aumento da susceptibilidade a outros estímulos, como a abrasão ou a atrição $0^{16}$. Isso resulta na remoção do tecido duro, que é direcionado para a cavidade oral ${ }^{14}$. Conforme o fator etiológico for agindo por mais tempo, maior será a lesão, podendo resultar em cavidades, conforme a dentina for se deteriorando ${ }^{4}$.

\section{Prevalência e incidência}

Na Tabela 1 podem ser observados os diversos estudos que avaliaram a presença de erosão dentária em crianças. A prevalência de lesões oriundas da erosão dentária é diversificada. De acordo com estudos previamente realizados, em crianças com dentição permanente, variou de $7,2 \%$ a $55.5 \%$, $13,8,12,13,17,18$. A maior prevalência encontrada foi nos jovens de 18 anos, no estudo de Okunseri et al. ${ }^{8}$ (2011), realizado nos Estados Unidos. Nos jovens de 13 a 15 anos avaliados no mesmo estudo, a prevalência foi $39,6 \%$, e nos jovens de 16 a 17 anos, foi de $44,5 \%$. A menor prevalência observada foi no estudo de Vargas Ferreira et al. ${ }^{18}$ (2011), realizado no Brasil, no qual $7,2 \%$ das crianças apresentaram erosão dentária ${ }^{18}$.

Poucos estudos são de desenho longitudinal. O estudo de El Aidi et al. ${ }^{19}$ (2011) observou uma prevalência de $32,2 \%$ no baseline, e após 1,5 ano de acompanhamento a prevalência foi de $42,2 \%$. Dugmore e Rock ${ }^{12}$ (2004) observaram que em crianças de 12 anos, a prevalência foi 56,3 após dois anos de acompanhamento a prevalência foi de $64,1 \%$. 
Tabela 1 - Estudos de base populacional que analisaram a erosão dentária em crianças e/ou adolescentes

\begin{tabular}{|c|c|c|c|c|c|c|c|c|}
\hline \multirow{2}{*}{ Autor e ano } & \multirow{2}{*}{ País } & \multirow{2}{*}{$\begin{array}{l}\text { Tipo de } \\
\text { estudo }\end{array}$} & \multirow{2}{*}{$\begin{array}{l}\text { Idade } \\
\text { (anos) }\end{array}$} & \multirow{2}{*}{$\mathrm{N}$} & \multirow{2}{*}{$\begin{array}{l}\text { Prevalência } \\
(\%)\end{array}$} & \multicolumn{3}{|c|}{ Distribuição } \\
\hline & & & & & & Dente & Superfície & Severidade \\
\hline Huew et al. ${ }^{20}$ (2011) & Líbia & Transversal & 12 & 791 & 40,8 & - & - & - \\
\hline $\begin{array}{l}\text { Okunseri et al. }{ }^{8} \\
(2011)\end{array}$ & USA & Transversal & $13-19$ & 1314 & $\begin{array}{l}\text { T: } 45 \\
\text { 18-19: } 56\end{array}$ & Incisivos centrais & Incisal & - \\
\hline $\begin{array}{l}\text { El Aidi et al. }{ }^{1}(2010) \text {, El } \\
\text { Aidi et al. }{ }^{19}(2011) \text { e El } \\
\text { Aidi et al. }{ }^{21}(2008)\end{array}$ & Holanda & Longitudinal & $10-12$ & 656 & $\begin{array}{l}\text { BL: } 32,2 \\
1,5 \text { anos: } 42,3 \\
11: 30,4 \\
15: 44,2\end{array}$ & $\begin{array}{l}\text { Primeiros } \\
\text { molares } \\
\text { inferiores }\end{array}$ & $\begin{array}{l}\text { Oclusal e } \\
\text { palatina }\end{array}$ & $\begin{array}{l}\text { BL: Esmalte } \\
\text { superficial } \\
\text { 1,5 anos: } \\
\text { dentina }\end{array}$ \\
\hline Wang et al..$^{9}(2010)$ & China & Transversal & $12-13$ & 1499 & $\begin{array}{l}\text { T: } 27.3 \\
12: 25,5 \\
13: 29,0\end{array}$ & Incisivos centrais & $\begin{array}{l}\text { Incisal e } \\
\text { oclusal }\end{array}$ & Esmalte \\
\hline $\begin{array}{l}\text { Vargas-Ferreira et al. }{ }^{18} \\
(2011)\end{array}$ & Brasil & Transversal & $11-14$ & 944 & 7.2 & $-(\mathrm{Al})$ & Palatina & Esmalte \\
\hline $\begin{array}{l}\text { Arnadottir et al. }{ }^{22} \\
(2010)\end{array}$ & Islândia & Transversal & $\begin{array}{l}6 \\
12 \\
15\end{array}$ & 2251 & $\begin{array}{l}\text { T: } 6 \\
12: 15,7 \\
15: 30,7\end{array}$ & $\begin{array}{l}\text { Primeiros } \\
\text { molares } \\
\text { inferiores }\end{array}$ & Palatina & Esmalte \\
\hline Bardolia et al. ${ }^{23}$ (2010) & UK & Transversal & $13-14$ & 629 & 20 & $\begin{array}{l}\text { Molares } \\
\text { superiores }\end{array}$ & Oclusais & Dentina \\
\hline $\begin{array}{l}\text { Gurgel et al. }{ }^{24} \\
(2011)\end{array}$ & Brasil & Transversal & $12-16$ & 414 & $\begin{array}{l}20 \\
12: 18 \\
16: 24\end{array}$ & $\begin{array}{l}\text { Incisivos } \\
\text { superiores }\end{array}$ & Vestibular & - \\
\hline $\begin{array}{l}\text { Correr et al. } \\
(2009)\end{array}$ & Brasil & Transversal & 12 & 389 & 26 & $\begin{array}{l}\text { Incisivos } \\
\text { superiores }\end{array}$ & Vestibular & Esmalte \\
\hline Mangueira et al. ${ }^{25}$ (2009) & Brasil & Transversal & $6-12$ & 983 & 38,2 & $-(\mathrm{Al})$ & Palatina & Esmalte \\
\hline Auad et al. ${ }^{10}(2007)$ & Brasil & Transversal & $13-14$ & 458 & 34,1 & $\begin{array}{l}\text { Incisivos } \\
\text { superiores }\end{array}$ & Palatina & Esmalte \\
\hline Peres et al. ${ }^{11}$ (2005) & Brasil & Transversal & 12 & 499 & 13,0 & $(\mathrm{Al})$ & Palatina & Esmalte \\
\hline $\begin{array}{l}\text { Milosevic et al. }(2004)^{26}, \\
\text { Bardsley et al. }(2004)^{27} \\
\text { (parte I-II) }\end{array}$ & UK & Transversal & 14 & 2351 & 53,5 & $\begin{array}{l}\text { Incisivos } \\
\text { centrais /molares } \\
\text { inferiores }\end{array}$ & $\begin{array}{l}\text { Oclusal e } \\
\text { incisal }\end{array}$ & $\begin{array}{l}\text { Dentina } \\
\text { superficial }\end{array}$ \\
\hline $\begin{array}{l}\text { Dugmore and Rock, } \\
2003,2004^{12,28}\end{array}$ & UK & Longitudinal & $\begin{array}{l}12 \\
14\end{array}$ & 1753 & $\begin{array}{l}\text { BL: } 12: 56,3 \\
\text { Após } 2 \text { anos } \\
14: 64,1\end{array}$ & $\begin{array}{l}\text { Incisivos } \\
\text { superficiais }\end{array}$ & Palatina & $\begin{array}{l}\text { Esmalte e } \\
\text { dentina }\end{array}$ \\
\hline $\begin{array}{l}\text { Truin et al. }{ }^{29} \\
(2005)\end{array}$ & Holanda & Transversal & $6-12$ & 832 & $\begin{array}{l}\text { 2002: } 24 \\
\text { 1998: } 3\end{array}$ & $\begin{array}{l}\text { Primeiros } \\
\text { molares dentes } \\
\text { anteriores }\end{array}$ & $\begin{array}{l}\text { Oclusal e } \\
\text { palatino }\end{array}$ & - \\
\hline Vanrijkon et al. ${ }^{13}$ (2002) & Holanda & Transversal & $\begin{array}{l}10-13 \\
15-16\end{array}$ & 745 & $\begin{array}{l}\text { 10-13: } 3,1 \\
15-16: 40,7\end{array}$ & $\begin{array}{l}\text { Primeiros } \\
\text { molares } \\
\text { inferiores }\end{array}$ & $\begin{array}{l}\text { Oclusal e } \\
\text { palatina }\end{array}$ & Esmalte \\
\hline $\begin{array}{l}\text { Al-Dlaigan et al. }{ }^{17}(2001) \\
\text { e Al-Dlaigan et al. }{ }^{30} \\
(2002) \text { (Parte I e II) }\end{array}$ & UK & Transversal & 14 & 1988 & 20,87 & Incisivos & Vestibular & $\begin{array}{l}\text { Esmalte e } \\
\text { dentina }\end{array}$ \\
\hline Künzel et al. ${ }^{31}$ (2000) & Cuba & Transversal & 12 & 1010 & 17,4 & $\begin{array}{l}\text { Incisivos centrais } \\
\text { superiores }\end{array}$ & Incisal & Esmalte \\
\hline Williams et al. ${ }^{5}$ (1999) & UK & Transversal & 14 & 525 & 24 & $-(\mathrm{Al})$ & Vestibular & Esmalte \\
\hline
\end{tabular}

BL: Baseline; T: Total; Al: Avaliação de incisivos 


\section{Distribuição}

Estudos mostram que os dentes mais comprometido com lesões erosivas são os incisivos centrais ${ }^{9,18}$. Outros estudos mostram que os primeiros molares inferiores estiveram mais frequentemente comprometidos com lesões erosivas ${ }^{1,9,13,29}$ (Tabela 1). Wiegand et al. ${ }^{32}$ (2006) constataram que a maior prevalência de erosão nos incisivos superiores deve-se ao fato de eles erupcionarem primeiro e se localizarem em área de grande contato com dieta ácida. Devido aos incisivos estarem localizados na frente da cavidade oral, estão predispostos à erosão por fatores extrínsecos ${ }^{9}$. Os incisivos inferiores podem não ser tão afetados devido à proteção de neutralização da saliva, excretada pelas glândulas salivares submandibular e sublingual ${ }^{25}$. Os molares, por sua vez, podem estar mais predispostos à erosão, devido à força oclusal durante a mastigação, que promoveria maior atrição dentária nos molares ${ }^{1,21}$.

\section{Severidade da erosão dentária}

A severidade das lesões pode variar, apresentando mudanças nas características superficiais do esmalte, perda de superfície do esmalte ${ }^{9,10,18,33}$ até a exposição de dentina $a^{7,12,23,26}$. A maioria de estudos constata que em crianças e adolescentes as lesões de erosão são confinadas ao esmalte ${ }^{3,11,18,19,25}$. Poucos estudos têm avaliado o desgaste erosivo em nível de dentina, encontrando lesões severas em crianças $^{17,26}$. Esse fato pode ser atribuído ao curto tempo de intervenção dos fatores etiológicos sob a estrutura dentária em crianças e adolescentes ${ }^{1,10,11,31}$.

\section{Superfícies}

Estudos apontam as superfícies palatinas como as mais comprometidas ${ }^{11,29}$. O efeito abrasivo da língua sobre o esmalte durante a fala e a deglutição podem contribuir para maior perda de tecido dental na área palatina, além disso, o efeito protetor da saliva nos dentes maxilares anteriores é diminuído, uma vez que a espessura da película salivar é menor nesse local ${ }^{33}$. De outro modo, a erosão na superfície palatina pode estar relacionada também à presença de ácidos gástricos, devido a refluxo gástrico involuntário (Gerd) ou voluntária por bulimia, que causam vômitos e regurgitação, devido ao efeito direto do ácido gástrico na região ${ }^{33,34}$.

Outros estudos constatam a face vestibular como a mais comprometida com erosão dentária ${ }^{7,11,12}$. Segundo Correr et al. ${ }^{7}$ (2009), a face vestibular está, geralmente, no caminho dos líquidos durante a ingestão, promovendo maior contato do líquido ácido com a vestibular ${ }^{7}$. Alguns fatores, como o acúmulo de substâncias ácidas nos incisivos superiores centrais e menor limpeza pela saliva, modo de ingestão de bebidas ácidas e distância das glândulas salivares e da língua, podem a maior frequência de lesões nessa face ${ }^{12}$.

A variação dos resultados entre esses estudos pode ser parcialmente explicada por diferenças nos critérios diagnósticos e índices usados, pois não existe um índice padrão de classificação da erosão dentária ${ }^{16}$. Variações relacionadas com os fatores socioeconômicos, comportamentais e culturais poderiam influenciar o resultado dos dados de prevalência ${ }^{9}$. A metodologia usada durante as avaliações e a obtenção de informações podem interferir nos resultados, provocando prevalências diversas. Assim, diversas faixas etárias podem influenciar os resultados ${ }^{35}$. A comparação entre os resultados dos estudos é difícil ${ }^{11}$.

O diagnóstico precoce possibilita a prevenção da condição ou do agravamento da lesão. Erosões iniciais são difíceis de visualizar, por isso o diagnóstico pode ser prejudicado. Assim é necessário que as superfícies dentárias estejam limpas, secas e bem iluminadas para ajudar no processo de diagnóstico ${ }^{3}$.

Um dos primeiros sinais da erosão é a descaracterização do esmalte, em que as linhas de deposição são perdidas ou atenuadas como reação à ação do ácido e do esmalte ${ }^{4}$. Sinais como perda do brilho do dente, ausência de placa macroscópica, polimento das superfícies atingidas pelos ácidos, perda de microanatomia podem sinalizar o distúrbio da erosão ${ }^{36}$ (Figura 1).

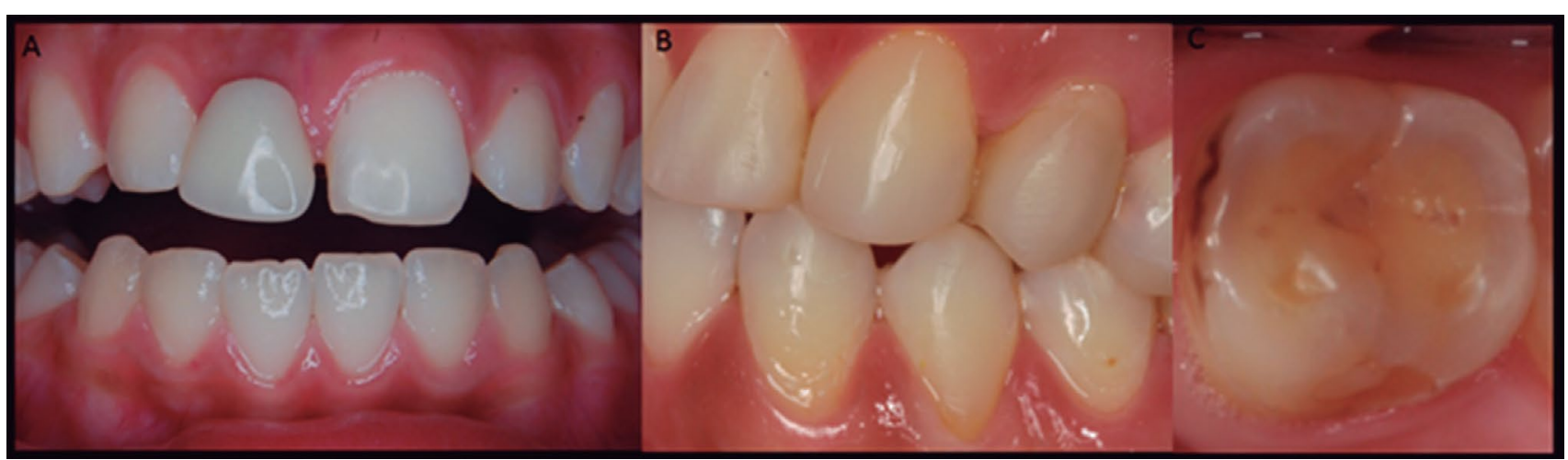

Figura 1 - Erosão dentária em adolescente. Em A: perda da anatomia vestibular do 21; em B: perda de estrutura na região cervical do 33 e 34 em C: cúspides com concavidades típicas de erosão dentária 
Quando o desgaste atinge a dentina, a progressão é mais rápida ${ }^{12}$. É característica a presença de um anel de esmalte cervical. Na oclusal de molares concavidades nas cúspides demonstram erosão ${ }^{4}$ (Figura 1-B). Se restaurações estão presentes, elas parecem "levantadas" por cima da estrutura dentária $^{15}$.

Com a progressão da perda de estrutura, aumenta a severidade da lesão, podendo se aproximar em profundidade à câmara pulpar ${ }^{3}$. Se o estímulo continuar, as sequelas que podem ser observadas incluem a erupção compensatória, formação de diastemas, alteração na dimensão vertical de oclusão, dor muscular e disfunção na articulação temporomandibular ${ }^{15}$.

Sendo a erosão uma condição multifatorial, várias condições podem se encontrar modulando a presença do desgaste dentário bem como condições clínicas, dieta, hábitos higiênicos, refluxo gastrointestinal e fatores econômicos e geográficos ${ }^{18}$.

\section{Fatores sociodemográficos}

\section{Nível socioeconômico}

A literatura aponta divergência entre os estudos, quando trata sobre a possibilidade de a erosão estar relacionada com o nível socioeconômico das crianças. Embora o nível socioeconômico não tenha demonstrado efeito total sobre a experiência de erosão, estudos demonstram que crianças economicamente favorecidas apresentaram significativamente menos erosão do que as de outros grupos ${ }^{12}$. Foi observado também que crianças em baixa posição socioeconômica tiveram maior prevalência de erosão ${ }^{1}$. Outros estudos não apontaram diferenças significativas ${ }^{18}$.

Uma das hipóteses que podem explicar essas diferentes constatações está relacionada ao acesso que as crianças têm a bebidas e comidas ácidas. Em alguns países, o acesso econômico a esses tipos de alimentos pode ser maior ${ }^{11}$, em outros países, o acesso econômico a esses alimentos pode ser mais restrito $^{12}$.

\section{Tipo de escola da criança}

O estudo de Peres et al. ${ }^{11}$ (2005) mostra que crianças de escolas particulares apresentaram maior prevalência de erosão ${ }^{11}$. No Brasil, tipo de escola é proxy de nível socioeconômico. Assim, essa situação pode estar relacionada com o acesso que as crianças têm a alimentos e bebidas ácidas ${ }^{11,25}$.

\section{Escolaridade dos pais}

O estudo de Wang et al. ${ }^{9}(2010)$, realizado com crianças de 12-13 anos, relacionou a situação socioeconômica das crianças baseada na ocupação e no grau de instrução dos pais com relação à presença de erosão. Ficou evidenciado que as crianças com mães com maior grau de instrução tendiam a ter menor prevalência de erosão dentária9 .

O grau de escolaridade dos pais influencia na medida em que os filhos dos pais com maior grau de instrução apresentam menor prevalência em relação aos filhos de pais com grau de instrução menor ${ }^{18}$. Pais com maior nível de educação podem ter maior acesso ao conhecimento sobre saúde bucal, como hábitos de higiene oral e dieta ${ }^{9}$.

\section{Idade}

Quanto maior a idade, maior a prevalência da erosão dentária ${ }^{12}$ e mais severa pode ser a lesão ${ }^{1}$. Estudos mostram que crianças de mais idade apresentam prevalência de erosão dentária maior ${ }^{1,12,18}$.

Com base nisso, pode-se dizer que o tempo de exposição pode ser um dos fatores cruciais para a ocorrência de erosão, tendo em vista que crianças mais velhas são expostas por mais tempo ao estímulo erosivo ${ }^{11}$.

\section{Sexo}

Há controvérsia quanto ao sexo das crianças estar associado com a presença de erosão. Adolescentes do sexo feminino foram observadas e apresentaram maior prevalência de erosão dentária ${ }^{9,37}$. No entanto, a maioria dos estudos mostra que crianças do sexo masculino apresentaram mais incidência do que meninas ${ }^{1,23}$.

De acordo com Bardolia et al. ${ }^{23}$ (2010), uma maior proporção de meninos teve dentina exposta em superfícies lisas / oclusais em relação às meninas, fornecendo, assim, apoio a resultados anteriores ${ }^{12,17,38}$. A força de mordida poderia explicar a diferença encontrada em prevalência de desgaste dentário erosivo entre meninos e meninas ${ }^{1}$. De outro modo, foi postulado como possível explicação para essa diferença que os meninos consomem mais frequentemente bebidas ácidas, se comparados às meninas $^{12,13}$

\section{Fatores comportamentais}

\section{Hábitos alimentares}

Hábitos alimentares podem influenciar na prevalência de erosão dentária. Estudos encontraram associação entre dieta ácida, como bebidas e comidas ácidas, e maior prevalência de erosão dentária ${ }^{1,8,9,12,20}$. Em crianças de 11 anos, da Holanda, a ingestão de bebidas carbonatadas foi associada à maior prevalência de erosão ${ }^{1}$. Em relação às crianças chinesas de 12 e 13 anos, também foi estudado como fator associado à erosão dentária a frequência do consumo de refrigerantes, também deixando 
clara a associação entre os fatores ${ }^{9}$. A frequência de consumo de bebidas ácidas açucaradas, como refrigerantes, e o tempo de consumo foram os principais fatores de risco estatisticamente significativos para a erosão dentária ${ }^{20}$. Porém, também houve autores que não encontraram relação entre erosão e hábitos alimentares ${ }^{13,18,33}$. Alguns autores encontraram uma associação entre o consumo frequente de bebidas carbonatadas e erosão dental ${ }^{22,39}$.

Mudanças nos estilos de vida, melhores condições financeiras e a disponibilidade de bebidas industrializadas potencialmente ácidas podem estar aumentando o consumo de refrigerantes e bebidas esportivas entre as crianças e os adolescentes ${ }^{40}$. Por outro lado, a intervenção da saliva como sustância tampão pode estar modulando a presença ou não de erosão dentária, mesmo com o alto consumo de alimentos ou bebidas ácidas ${ }^{7}$.

\section{Hábitos de higiene}

Escovar os dentes duas vezes por dia aumentou as chances de erosão em superfícies lisas / oclusais $^{23,38}$. A escovação dentária também pode influenciar a progressão de lesões erosivas ${ }^{41}$. Procedimentos abrasivos podem remover da superfície frágil tecidos duros dentais desmineralizados. Desse modo, o momento de escovação após um ataque erosivo bem como o tipo de escova e de pasta de dentes utilizadas podem influenciar a progressão de desgaste dental ${ }^{41}$.

\section{Consumos de medicamentos ou vitaminas}

Medicamentos, como ácido acetil-salicílico e drogas antiasmáticas, têm associação direta com a erosão dentária por terem baixo $\mathrm{pH}$, o que promove a dissolução direta dos tecidos dentários duros ${ }^{42}$. Assim como esses, pelo mesmo motivo, o consumo exagerado de vitamina $\mathrm{C}$, em tabletes, também pode ser considerado um dos fatores favoráveis ao surgimento de lesões erosivas ${ }^{1}$.

A erosão dentária associada ao uso de medicamentos asmáticos pode ser primariamente atribuído ao fato de que a maioria desses medicamentos são ácidos e, portanto, direta ameaça erosiva à dentição. Além disso, diminuem o tamponamento salivar e a capacidade e o fluxo ${ }^{3}$. $\mathrm{O}$ frequente uso de tais medicações é seguido pelo consumo de bebidas ácidas, para compensar a secura bucal e superar o gosto amargo do fármaco. Ademais, condições médicas, tais como vômitos, azia e problemas gástri$\cos$, foram mais comumente relatados em pacientes asmáticos, contribuindo, assim, para $\mathrm{ED}^{43}$.

\section{Esportes}

A natação foi abordada como um fator associado à erosão dentária. Ficou evidenciado que crianças que praticavam natação, que tinham mais contato com o gás cloro da piscina, apresentaram maior prevalência em relação aos nadadores amadores e não profissionais ${ }^{4}$. A causa pode ser o monitoramento inadequado das piscinas, que pode baixar o $\mathrm{pH}$ da água, consequentemente aumentando a possibilidade de lesões. A saliva não alcançaria a ação protetora devido ao cloro ácido ${ }^{44}$.

\section{Fatores biológicos}

\section{Cárie dentária}

Crianças que apresentam cárie podem ser mais propensas a ter erosão dentária ${ }^{12,35}$. A maior margem significante de pontuação média de CPO-D foi encontrada em crianças com exposição de dentina, que é semelhante a um relatório de uma associação positiva $(\mathrm{OR}=1,5)$ entre cárie e erosão, tanto nos grupos de idade menor quanto nos de 14 anos de idade $^{12}$.

Como ambas as doenças são causadas por ácidos, é provável que condições bucais que favorecem uma doença também favorecem a outra. Por outro lado, a perda de elementos de proteção, tais como a saliva, pode ser um fator de risco para ambas as doenças ${ }^{3}$. Em geral, superfícies propensas à erosão (pontas de cúspide e superfícies palatais) ou a atrito (bordas incisais) não são aquelas em que ocorre a cárie ${ }^{23}$.

\section{Defeitos de esmalte}

Crianças com hipoplasia do esmalte estão propensas a ter também uma forte erosão dentária na dentição permanente, e os dentes permanentes avaliados individualmente, com erosão, apresentaram maior propensão à hipoplasia do esmalte de acordo com o estudo de Kazoullis et al. ${ }^{35}$ (2007). Os autores $^{35}$ postularam que a mineralização reduzida ou alterada observada em defeitos de esmalte pode levar a uma maior facilidade de dissolução por ácidos e a perda de estrutura do dente por atrito e abrasão.

\section{Mal oclusão}

No estudo de Dugmore e $\operatorname{Rock}^{12}$ (2004), ficou constatado que ter uma anomalia oclusal diminuiu as chances de ocorrer erosão em crianças com 12 anos. Uma redução da erosão pode possivelmente ser resultado de dentes em um arco não alinhado, o que diminui a taxa de purificação e aumenta a distribuição de saliva ao redor dos dentes, potencializando ou diminuindo os seus efeitos protetores ${ }^{12}$. 


\section{Vômitos ou problemas gástricos}

O número de pacientes com problemas gástricos tem aumentado com o estresse da vida moderna ${ }^{45}$. Essas doenças induzem o contato do suco gástrico com a cavidade oral. Devido ao seu caráter ácido, sua presença na cavidade oral pode resultar em erosão dentária. Pacientes com esses distúrbios tendem a ter redução na capacidade do tampão salivar, o que pode aumentar o potencial de erosão dos ácidos gástricos ${ }^{46}$.

\section{Condições médicas}

Crianças asmáticas podem ser mais propensas a desenvolver lesões erosivas ${ }^{17,47}$. Essa propensão deve-se aos medicamentos asmáticos, que podem reduzir a proteção salivar contra ácidos extrínsecos ou intrínsecos, facilitando, assim, a ocorrência de erosão dentária ${ }^{33,47}$.

\section{Discussão}

A erosão é uma condição dentária que tem sido discutida e estudada nos últimos anos ${ }^{18,22,23}$. A queda da prevalência da cárie dentária foi observada em alguns países, especialmente europeus, tem promovido maior visualização da erosão dentária. O número de estudos que pesquisam a erosão dentária desde então foi aumentando e, devido a esse motivo, a percepção de maior prevalência dessa condição tem sido verificada ${ }^{12,26}$.

A literatura indica a existência de estudos epidemiológicos transversais de base populacional, mostrando que a prevalência de erosão em crianças varia entre $7,2 \%$ a $55,5 \% \%^{1,3,8,12,13,17,18}$. Um estudo realizado na Arábia Saudita encontrou uma prevalência de $90 \%$, considerada extremamente eleva$\mathrm{da}^{48}$. Porém, a análise de estudos transversais não permite visualizar se a erosão dentária de fato está aumentando.

Poucos estudos longitudinais avaliaram a erosão. Dugmore e Rock ${ }^{12}$ (2004) e EL Aidi et al. ${ }^{1}$ (2010) acompanharam crianças e adolescentes por 2 a 3 anos e permitiram elucidar parcialmente essa incógnita. Dugmore e Rock ${ }^{12}$ (2004) observaram que, dos 12 aos 14 anos, houve um aumento da incidência de erosão. Já El Aidi et al. ${ }^{1}$ (2010) encontrou aumento da erosão dentária até o segundo ano de avaliação e, após esse período, ocorreu uma estabilização. Observou-se, ainda, tanto nos estudos de prevalência quanto nos de incidência, que a severidade das lesões nessa população foi limitada ao esmalte $^{7,18,19}$. Com o tempo, essas lesões podem alcançar a dentina, de forma que crianças mais velhas tendem a apresentar lesões mais avançadas e com maior severidade $^{1,23,26}$.

A dificuldade em poder avaliar e determinar se a erosão dentária está aumentando tem sido discu- tida por diversos motivos, que incluem a natureza multifatorial da erosão dentária e a dificuldade de padronização da metodologia utilizada, assim como dificuldades diagnósticas da erosão ${ }^{18}$.

A forma de medida de erosão pode representar um fator relacionado com a variabilidade das prevalências observadas. Não existe um índice padrão ouro que possa ser usado, sendo assim, os estudos não seguem critérios para seleção entre os diversos índices existentes. Já há alguns anos, o Bewe é o índice recomendado pela comunidade odontológica para servir como padrão ouro, porém esse índice, até o momento, tem sido pouco utilizado ${ }^{49}$.

A avaliação de alguns dentes ou de todos os dentes tem sido um fator discutido e que poderia também influenciar nos resultados. Estudos ${ }^{1,19}$ têm usado dentes índices, pois os resultados permitem observar que os dentes mais atingidos seriam os dentes anterossuperiores e os molares. Parece adequado avaliar esses dentes, diminuindo o risco de estimação e subestimação, já que a inclusão de outros dentes poderia também promover a ocorrência de outros tipos de desgaste. Devido ao caráter multifatorial da erosão dentária, a influência de outros fatores que estariam modulando a presença da erosão também poderiam determinar a variedade de resultados.

A situação socioeconômica e a sua influência no aparecimento de lesões erosivas podem indicar a estreita relação entre dieta e acesso a certos alimentos e comidas, assim como o acesso à informação e aos cuidados em relação à condição dentária ${ }^{50}$. Dependendo do contexto social e cultural, uma criança ou um adolescente podem ter condições de adquirir e consumir com maior frequência alimentos ácidos ou adquirir e consumir alimentos saudáveis e ter uma dieta antierosiva. Devido a essa situação, em alguns países, crianças com melhores situações socioeconômicas podem estar em maior risco de apresentar erosão dentária, ou aquelas economicamente desfavoráveis podem estar com risco reduzido.

O consumo elevado de alimentos ácidos na dieta pode promover maior erosão ${ }^{51}$. Bebidas, como refrigerantes e bebidas esportivas, alguns alimentos, como molhos, lanches, salgados, ou um consumo acentuado de certos medicamentos estão associados à maior prevalência de erosão $0^{31,52}$, porém, outros estudos não encontraram essa associação $0^{18,33}$. Outras condições, como o maior consumo de alimentos lácteos, como leite, queijos ou iogurte, o fluxo ou a capacidade tampão da saliva, a presença de flúor no meio oral e a escovação dentária acentuada, podem modular a presença da erosão dentária ${ }^{53}$. Em uma recente meta-análise, Salas et al. ${ }^{54}$ (2015) verificaram que componentes da dieta (refrigerantes, lanches, doces ácidos e sucos naturais ácidos) aumentaram a ocorrência de erosão, enquanto que o leite e iogurte tiveram efeito protetor. Os autores também concluíram que os métodos para mensurar 
o consumo de bebidas / alimentos podem influenciar a heterogeneidade dos estudos.

A prevalência entre os estudos é variável ${ }^{18,26}$, de baixa severidade, e pode estar influenciada por diversos fatores ${ }^{54}$. Apesar de a erosão não representar um problema de saúde pública, ela é uma condição que, se não controlada, pode progredir e levar a perdas importantes de estrutura dentária. A partir do conhecimento obtido por meio das diversas pesquisas, as populações de risco podem ser identificadas e, dessa forma, medidas preventivas aplicadas em estágios iniciais podem cessar a progressão da erosão, reduzindo, assim, a necessidade de futuras intervenções.

\section{Considerações finais}

A prevalência da erosão dentária em crianças com dentes permanentes varia, podendo ser baixa, intermediária ou alta. O diagnóstico clínico da lesão erosiva pode ser confundido com outros tipos de desgastes. Existem fatores sociodemográficos, comportamentais e biológicos associados à erosão dentária.

\section{Abstract}

Objective: this study reviewed epidemiological studies conducted with children 8-19 yo with permanent dentition, without any orthodontic treatment or systemic problems, and that assessed possible factors commonly associated with tooth erosion. Literature review: dental erosion is the loss of hard tissue from dental surface by chemical influence with no bacteria involvement. Dental erosion is a multifactorial oral condition associated with extrinsic factors, such as intake of carbonated acidic drinks, or intrinsic factors, such as the association to regurgitation episodes, resulting in demineralization of the tooth surface. Erosive wear is commonly associated with other types of tooth wear of different etiology. If not controlled, dental erosion can promote esthetic, functional and/or occlusion problems. Final considerations: the prevalence of dental erosion in children with permanent dentition ranged from $7,2 \%$ to $55.5 \%$. High prevalence of dental erosion was associated with older children and higher consumption of some acidic foods such as soft drinks, as well as lower consumption of some lacteous food, such as milk. In children and adolescents, the severity of the lesions was limited to the enamel. The association with socioeconomic and biological factors showed diverse results.

Keywords: Dental erosion. Child. Adolescents. Prevalence. Associated factors.

\section{Referências}

1. El Aidi H, Bronkhorst EM, Huysmans MC, Truin GJ. Dynamics of tooth erosion in adolescents: a 3-year longitudinal study. J Dent 2010; 38(2):131-7.
2. Jaeggi T, Lussi A. Prevalence, incidence and distribution of erosion. Monogr Oral Sci 2006; 20:44-65.

3. Nahas Pires Correa MS, Nahas Pires Correa F, Nahas Pires Correa JP, Murakami C, Mendes FM. Prevalence and associated factors of dental erosion in children and adolescents of a private dental practice. Int $\mathrm{J}$ Paediatr Dent 2011; 21(6):451-8.

4. Lussi A. Erosive tooth wear - a multifactorial condition of growing concern and increasing knowledge. Monogr Oral Sci 2006; 20:1-8.

5. Williams D, Croucher R, Marcenes W, O'Farrell M. The prevalence of dental erosion in the maxillary incisors of 14-year-old schoolchildren living in Tower Hamlets and Hackney, London, UK. Int Dent J 1999; 49(4):211-6.

6. Taji S, Seow WK. A literature review of dental erosion in children. Aust Dent J 2010; 55(4):358-67; quiz 475.

7. Correr GM, Alonso RC, Correa MA, Campos EA, Baratto-Filho F, Puppin-Rontani RM. Influence of diet and salivary characteristics on the prevalence of dental erosion among 12-year-old schoolchildren. J Dent Child 2009; 76(3):181-7.

8. Okunseri C, Okunseri E, Gonzalez C, Visotcky A, Szabo A. Erosive tooth wear and consumption of beverages among children in the United States. Caries Res 2011; 45(2):130-5.

9. Wang P, Lin HC, Chen JH, Liang HY. The prevalence of dental erosion and associated risk factors in 12-13-year-old school children in Southern China. BMC Public Health 2010; 10:478.

10. Auad SM, Waterhouse PJ, Nunn JH, Steen N, Moynihan PJ. Dental erosion amongst 13- and 14-year-old Brazilian schoolchildren. Inter Dent J 2007; 57(3):161-7.

11. Peres KG, Armenio MF, Peres MA, Traebert J, De Lacerda JT. Dental erosion in 12-year-old schoolchildren: a cross-sectional study in Southern Brazil. Int J Paediatr Dent 2005; 15(4):249-55.

12. Dugmore CR, Rock WP. The prevalence of tooth erosion in 12-year-old children. Br Dent J 2004; 196(5):279-82; discussion 3.

13. Van Rijkom HM, Truin GJ, Frencken JE, Konig KG, van 't Hof MA, Bronkhorst EM, et al. Prevalence, distribution and background variables of smooth-bordered tooth wear in teenagers in the hague, the Netherlands. Caries Res 2002; 36(2):147-54.

14. Lussi A, Schlueter N, Rakhmatullina E, Ganss C. Dental erosion--an overview with emphasis on chemical and histopathological aspects. Caries Res 2011; 45 Suppl 1:2-12.

15. Donovan T. Dental erosion. J Esthet Restor Dent 2009; 21(6):359-64.

16. Ganss C, Young A, Lussi A. Tooth wear and erosion: methodological issues in epidemiological and public health resear$\mathrm{ch}$ and the future research agenda. Community Dental Health 2011; 28(3):191-5.

17. Al-Dlaigan YH, Shaw L, Smith A. Dental erosion in a group of British 14-year-old, school children. Part I: Prevalence and influence of differing socioeconomic backgrounds. Br Dent J $2001 ; 190(3): 145-9$.

18. Vargas-Ferreira F, Praetzel JR, Ardenghi TM. Prevalence of tooth erosion and associated factors in 11-14-year-old Brazilian schoolchildren. J Public Health Dent 2011; 71(1):6-12.

19. El Aidi H, Bronkhorst EM, Huysmans MC, Truin GJ. Multifactorial analysis of factors associated with the incidence and progression of erosive tooth wear. Caries Res 2011; 45(3):303-12. 
20. Huew R, Waterhouse PJ, Moynihan PJ, Kometa S, Maguire A. Dental erosion and its association with diet in Libyan schoolchildren. Eur Arch Paediatr Dent 2011; 12(5):234-40.

21. El Aidi H, Bronkhorst EM, Truin GJ. A longitudinal study of tooth erosion in adolescents. J Dental Res 2008; 87(8):731-5.

22. Arnadottir IB, Holbrook WP, Eggertsson H, Gudmundsdottir $\mathrm{H}$, Jonsson SH, Gudlaugsson JO, et al. Prevalence of dental erosion in children: a national survey. Community Dent Oral Epidemiol 2010; 38(6):521-6.

23. Bardolia P, Burnside G, Ashcroft A, Milosevic A, Goodfellow SA, Rolfe EA, et al. Prevalence and risk indicators of erosion in thirteen- to fourteen-year-olds on the Isle of Man. Caries Res 2010; 44(2):165-8.

24. Gurgel CV, Rios D, Buzalaf MA, da Silva SM, Araujo JJ, Pauletto AR, et al. Dental erosion in a group of 12 - and 16-year-old Brazilian schoolchildren. Pediatr Dent 2011; 33(1):23-8.

25. Mangueira DF, Sampaio FC, Oliveira AF. Association between socioeconomic factors and dental erosion in Brazilian schoolchildren. J Public Health Dent 2009; 69(4):254-9.

26. Milosevic A, Bardsley PF, Taylor S. Epidemiological studies of tooth wear and dental erosion in 14-year old children in North West England. Part 2: The association of diet and habits. Br Dent J 2004; 197(8):479-83; discussion 3; quiz 505.

27. Bardsley PF, Taylor S, Milosevic A. Epidemiological studies of tooth wear and dental erosion in 14-year-old children in North West England. Part 1: The relationship with water fluoridation and social deprivation. Br Dent J 2004; 197(7):4136; discussion 399.

28. Dugmore CR, Rock WP. The progression of tooth erosion in a cohort of adolescents of mixed ethnicity. Int J Paediatr Dent 2003; 13(5):295-303.

29. Truin GJ, van Rijkom HM, Mulder J, van't Hof MA. Caries trends 1996-2002 among 6- and 12-year-old children and erosive wear prevalence among 12-year-old children in The Hague. Caries Res 2005; 39(1):2-8.

30. Al-Dlaigan YH, Shaw L, Smith AJ. Dental erosion in a group of British 14-year-old, school children. Part III: Influence of oral hygiene practises. Br Dent J 2002; 192(9):526-30.

31. Kunzel W, Cruz MS, Fischer T. Dental erosion in Cuban children associated with excessive consumption of oranges. Eur J Oral Sci 2000; 108(2):104-9.

32. Wiegand A, Muller J, Werner C, Attin T. Prevalence of erosive tooth wear and associated risk factors in 2-7-year-old German kindergarten children. Oral Dis 2006; 12(2):117-24.

33. Gurgel CV, Rios D, de Oliveira TM, Tessarolli V, Carvalho FP, Machado MA. Risk factors for dental erosion in a group of 12- and 16-year-old Brazilian schoolchildren. Int J Paediatr Dent 2011; 21(1):50-7.

34. Brewerton TD. Bulimia in children and adolescents. Child Adolesc Psychiatr Clin N Am 2002; 11(2):237-56, viii.

35. Kazoullis S, Seow WK, Holcombe T, Newman B, Ford D. Common dental conditions associated with dental erosion in schoolchildren in Australia. Pediatr Dent 2007; 29(1):33-9.

36. Ganss C, Lussi A. Diagnosis of erosive tooth wear. Monogr Oral Sci 2006; 20:32-43.

37. Shih PC, Hung YC, Chen YL, Tsai HJ, Chen CY, Huang CJ. Agreement of cardiac output measurement between pulse contour analysis and thermodilution in various body positions: a porcine study. J Surg Res 2013; 181(2):315-22.

38. Al-Dlaigan YH, Shaw L, Smith A. Dental erosion in a group of British 14-year-old school children. Part II: Influence of dietary intake. Br Dent J 2001; 190(5):258-61.

39. Bartlett DW, Coward PY, Nikkah C, Wilson RF. The prevalence of tooth wear in a cluster sample of adolescent scho- olchildren and its relationship with potential explanatory factors. Br Dent J 1998; 184(3):125-9.

40. Vartanian LR, Schwartz MB, Brownell KD. Effects of soft drink consumption on nutrition and health: a systematic review and meta-analysis. Am J Public Health 2007; 97(4):66775 .

41. Ganss C, Schlueter N, Hardt M, von Hinckeldey J, Klimek J. Effects of toothbrushing on eroded dentine. Eur J Oral Sci 2007;115(5):390-6.

42. Tredwin CJ, Scully C, Bagan-Sebastian JV. Drug-induced disorders of teeth. J Dent Res 2005; 84(7):596-602.

43. Hamasha AA, Zawaideh FI, Al-Hadithy RT. Risk indicators associated with dental erosion among Jordanian school children aged 12-14 years of age. Int J Paediatr Dent 2014; 24(1):56-68.

44. Zero DT, Lussi A. Behavioral factors. Monogr Oral Sci 2006; 20:100-5.

45. Ranjitkar S, Kaidonis JA, Smales RJ. Gastroesophageal reflux disease and tooth erosion. Int J Dent 2012; 2012:479850.

46. Dahshan A, Patel H, Delaney J, Wuerth A, Thomas R, Tolia V. Gastroesophageal reflux disease and dental erosion in children. J Pediatr 2002; 140(4):474-8.

47. Manuel ST, Kundabala M, Shetty N, Parolia A. Asthma and dental erosion. Kathmandu Univ Med J 2008; 6(23):370-4.

48. Al-Zarea BK. Tooth surface loss and associated risk factors in northern saudi arabia. ISRN Dent 2012; 2012:161565.

49. Olley RC, Wilson R, Bartlett D, Moazzez R. Validation of the Basic Erosive Wear Examination. Caries Res 2014; 48(1):51-6.

50. Carvalho TS, Lussi A, Jaeggi T, Gambon DL. Erosive tooth wear in children. Monogr Oral Sci 2014; 25:262-78.

51. Lussi A, Carvalho TS. Erosive tooth wear: a multifactorial condition of growing concern and increasing knowledge. Monogr Oral Sci 2014; 25:1-15.

52. Lussi A, Hellwig E, Zero D, Jaeggi T. Erosive tooth wear: diagnosis, risk factors and prevention. Am J Dent 2006; 19(6):319-25.

53. Barbour ME, Lussi A. Erosion in relation to nutrition and the environment. Monogr Oral Sci 2014; 25:143-54.

54. Salas MM, Nascimento GG, Huysmans MC, Demarco FF. Estimated prevalence of erosive tooth wear in permanent teeth of children and adolescents: an epidemiological systematic review and meta-regression analysis. J Dent 2015; 43(1):42-50.

\section{Endereço para correspondência:}

Flávio Fernando Demarco

Universidade Federal de Pelotas, Faculdade de

Odontologia, Rio Grande do Sul, Brasil

Endereço: Rua Gonçalves Chaves, 457

CEP 96015560, RS

Telefone: (53) 3222-4439

E-mail: flavio.demarco@pq.cnpq.br ffdemarco@gmail.com

Recebido: 26 / 11/2014. Aceite: 22 /06 / 2015 